\title{
Studienerfahrungen und Karrierepläne angehender Ärztinnen und Ärzte
}

\author{
B. Buddeberg-Fischer ${ }^{a}$, R. Klaghofer ${ }^{a}$, E. Vetsch ${ }^{a}$, T. Abel ${ }^{b}$, C. Buddeberg ${ }^{a}$
}

\section{Ausgangslage}

Seit Mitte der 90er Jahre studieren in den westlichen Ländern mehr Frauen als Männer Medizin. Medizinstudentinnen sind gleich erfolgreich wie ihre männlichen Kollegen [1]. Nach Studienabschluss durchläuft eine immer grössere Zahl der jungen Ärztinnen und Ärzte eine fachärztliche Weiterbildung. Im Jahr 2001 hatten in der Schweiz 50\% aller berufstätigen Ärztinnen und 81,4\% aller Ärzte einen Facharzttitel [2]. Allerdings zeigt sich bei der Wahl der Spezialisierung eine geschlechtsabhängige Verteilung. Frauen wählen vorzugsweise die Fachgebiete Allgemeinund Innere Medizin, Gynäkologie/Geburtshilfe, Pädiatrie und Psychiatrie, Männer sind in operativen und technisch-apparativen Fächern stärker vertreten. Im Vergleich $\mathrm{zu}$ früheren Jahren zeichnet sich jedoch eine breitere Streuung des Frauenanteils in den verschiedenen Spezialgebieten $a b$.

Wie die Geschlechterverteilung bei den Studienanfängern zeigt, nimmt das Interesse der Männer am Arztberuf ab, während immer mehr Frauen die Medizin als ein für sie attraktives Berufsfeld entdecken. Diese Entwicklung, die man als «Feminisierung der Medizin» [3] charakterisieren kann, bringt auch Veränderungen im Hinblick auf Karriereentwicklungen mit sich. Wie neuere Untersuchungen zeigen, haben angehende Ärztinnen andere Vorstellungen über ihre Berufslaufbahn als angehende Ärzte [4]. Obwohl Medizinerinnen eine ähnlich hohe Berufsmotivation haben wie ihre männlichen Kollegen, priorisieren sie nach wie vor die Gründung einer eigenen Familie vor einer beruflichen Karriere [5]. Ärzte wünschen sich in der Mehrzahl zwar ebenfalls eine Familie, dies hindert sie jedoch nicht an ihrer Karriereplanung [6].

Bisherige Studien, welche Determinanten für die unterschiedlichen Karrierewege von Ärztinnen und Ärzten untersuchten, beschränkten sich auf Querschnittuntersuchungen. Umfassendere Längsschnittstudien, in denen individuelle, institutionelle und gesellschaftliche Determinanten der Karriereentwicklung berücksichtigt werden, gibt es bisher erst wenige [6-8]. Aus der Schweiz liegen noch keine entsprechenden Untersuchungen vor. Dies war u.a. der Anlass für eine prospektive Verlaufsstudie zur beruflichen Entwicklung von jungen Deutschschweizer Ärztinnen und Ärzten. Im Rahmen eines vom Schweizerischen Nationalfonds geförderten Forschungsprojektes wurden Studierende der Humanmedizin der drei Deutschschweizer Medizinischen Fakultäten Basel, Bern und Zürich des 6. Studienjahres im Herbst 2000 (Kohorte A) und Herbst 2001 (Kohorte B) zu ihren Studienerfahrungen und Karriereplänen befragt. Die Studie soll während der Weiterbildungszeit der Teilnehmenden über einen Zeitraum von 6 bis 8 Jahren fortgesetzt werden. Im folgenden wird über einige wichtige Ergebnisse der Ersterhebung berichtet.

\section{Studiendesign und Teilnehmende}

In einem ausführlichen Fragebogen wurden die Studierenden gebeten, Angaben $\mathrm{zu}$ folgenden Themenbereichen zu machen: Soziodemographische Merkmale, Studien- und Dissertationserfahrungen, Laufbahnplanung, berufliche Motivation, Persönlichkeitseigenschaften, Lebenszufriedenheit und Wohlbefinden. Da die Ergebnisse aus den beiden Kohorten A und B nur geringfügige Unterschiede zeigten, werden die Ergebnisse für beide Kohorten zusammen dargestellt.

Von den 1004 immatrikulierten und angeschriebenen Studierenden der Humanmedizin der Universitäten Basel, Bern und Zürich haben 703 Personen an der Befragung teilgenommen (Rücklaufquote $70 \%$ !). Davon sind 370 (52,6\%) Frauen und 333 (47,4\%) Männer.

\section{Soziodemographische Angaben}

Das durchschnittliche Alter liegt bei 27,3 Jahren (SD 2,34 Jahre, Streubreite 23-44 Jahre). Die Mehrzahl ist ledig und kinderlos. Von 42,6\% der Befragten hat mindestens ein Elternteil eine akademische Ausbildung. 13,7\% der Studierenden kommen aus einem Elternhaus, in welchem ein Elternteil Medizin studiert hat; nur bei $0,7 \%$ ist dies die Mutter, bei 11,0\% der Vater. Bei 2\% der Befragten sind beide Eltern Ärzte. 
Zur Gewährleistung der Anonymität der Befragten sind die Fragebogen nur mit einem Code versehen. Die Teilnehmenden schickten ihre aktuelle Adresse getrennt vom Fragebogen an eine unabhängige Adressverwaltungsstelle der Universität Bern, so dass sie für die Rückmeldungen einiger Ergebnisse der Erstbefragung und für die geplanten weiteren Erhebungen kontaktiert werden können.

\section{Studien- und Dissertationserfahrung}

Klinische Erfahrungen im Studium (Abb. 1)

Die Studierenden wurden nach den klinischen Erfahrungen während der Unterassistenzstellen/ Blockkurse gefragt. Die vorgegebenen Antwortalternativen reichen von 1 «sehr selten» bis 4 «sehr oft». Für die Auswertung wurde für jede Frage ein Mittelwert über alle Unterassistenzstellen/Blockkurse berechnet.

Die Befragten machten Angaben, ob ihnen in der Patientenversorgung verantwortungsvolle Aufgaben zugeteilt wurden, ob Vorgesetzte ihre Arbeit lobten und wie die fachliche Anleitung war. Diese drei Aspekte in der klinischen Arbeit wurden als gut beurteilt. Der persönliche Kontakt mit den betreuenden Assistierenden wurde mehrheitlich als sehr gut eingeschätzt.

\section{Abbildung 1}

Erfahrungen im Studium während Unterassistenzstellen bzw. Blockkursen (Rating: 1 «sehr selten» bis 4 «sehr oft»).

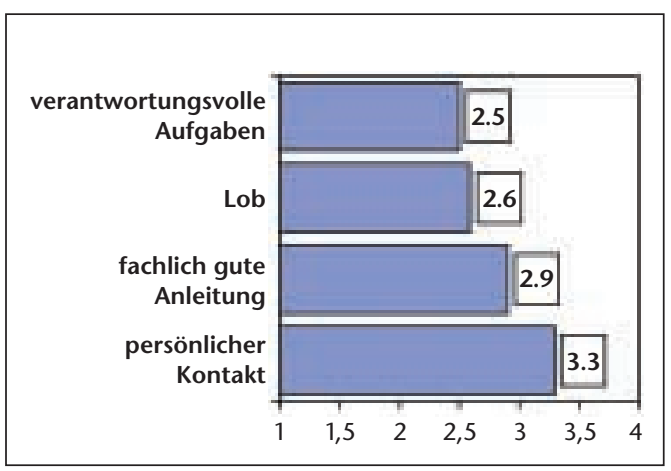

\section{Dissertation}

$21,5 \%$ der Befragten haben ihre Dissertation bereits abgeschlossen. 54,8\% arbeiten zur Zeit der Befragung an der Dissertation, weitere 20,4\% haben ihre Dissertation noch nicht begonnen, 3,3\% haben sie abgebrochen. Die Frage, ob sie vor ihrer jetzigen eine Dissertation abgebrochen haben, beantworten weitere 5,7\% mit ja. Insgesamt geben 9,0\% aller Teilnehmenden an, je eine Dissertation abgebrochen zu haben. Mit der Betreuung sind die Dissertierenden mehrheitlich eher bzw. sehr zufrieden (73,8\%).

\section{Tabelle 1}

Aspekte bei der Wahl des Fachgebietes.

\begin{tabular}{|c|c|c|}
\hline Wie wichtig sind für Sie folgende Aspekte bei der Wahl des Fachgebietes? & wichtig \% & Geschlechtsunterschied \\
\hline kurze Ausbildungsdauer & 36,5 & $\mathrm{~F}>\mathrm{M}^{* * *}$ \\
\hline gute Vereinbarkeit mit einer eigenen Familie & 83,4 & \\
\hline spätere Selbständigkeit & 61,0 & \\
\hline niedrige Praxisinvestitionen im Falle einer späteren Selbständigkeit & 24,8 & \\
\hline intensiver Patientenkontakt & 87,3 & $\mathrm{~F}>\mathrm{M}^{* *}$ \\
\hline manuell-operative Tätigkeit & 59,1 & $M>F^{*}$ \\
\hline technisch-apparative Tätigkeit & 31,4 & $\mathrm{M}>\mathrm{F}^{* * *}$ \\
\hline Forschungstätigkeit & 25,0 & $\mathrm{M}>\mathrm{F}^{\star * *}$ \\
\hline Vielseitigkeit des Fachgebietes & 96,1 & \\
\hline Überschaubarkeit des Fachgebietes & 63,4 & $\mathrm{~F}>\mathrm{M}^{* * *}$ \\
\hline positive Erfahrungen während des Studiums & 85,9 & \\
\hline spätere Übernahme einer Arztpraxis von Verwandten & 4,3 & \\
\hline hohes Einkommen & 25,3 & $\mathrm{M}>\mathrm{F}^{* * *}$ \\
\hline hohes Ansehen & 19,1 & $M>F^{* *}$ \\
\hline anderer Aspekt, nämlich & 91,9 & \\
\hline
\end{tabular}




\section{Laufbahnplanung}

Vereinbarte Weiterbildungsstellen

Von den Teilnehmenden haben 44,5\% zum Zeitpunkt der Befragung schon eine oder mehrere Assistenzstellen fest vereinbart.

\section{Wahl der beruflichen Richtung}

$89,4 \%$ der Befragten streben nach dem Staatsexamen eine fachärztliche Weiterbildung (FMH bzw. neu «Eidgenössischer Weiterbildungstitel») an, andere Möglichkeiten, wie z. B. eine Tätigkeit in der Industrie, Gesundheitsökonomie, im EDVund E-Health-Bereich oder im Journalismus, werden nur vereinzelt genannt. Lediglich 5,0\% äussern, sich noch nicht entschieden zu haben, welchen beruflichen Weg sie einschlagen wollen.

\section{Kriterien für die Wahl des Fachgebietes}

Wie aus Tabelle $1 \mathrm{zu}$ sehen ist, erachten mehr als vier Fünftel der angehenden Ärztinnen und Ärzte die Aspekte «Vielseitigkeit des Fachgebietes», «intensiver Patientenkontakt», «positive Erfahrungen während des Studiums» und «gute Vereinbarkeit mit einer eigenen Familie» bei der Wahl des Fachgebietes als wichtig. Bezüglich der Gewichtung einzelner Aspekte für die Wahl des Fachgebietes zeigen sich signifikante ge-

\section{Abbildung 2}

Einschätzung der beruflichen Motivation auf den drei Skalen: intrinsische und extrinsische Motivation sowie extraprofessionale Belange [9].

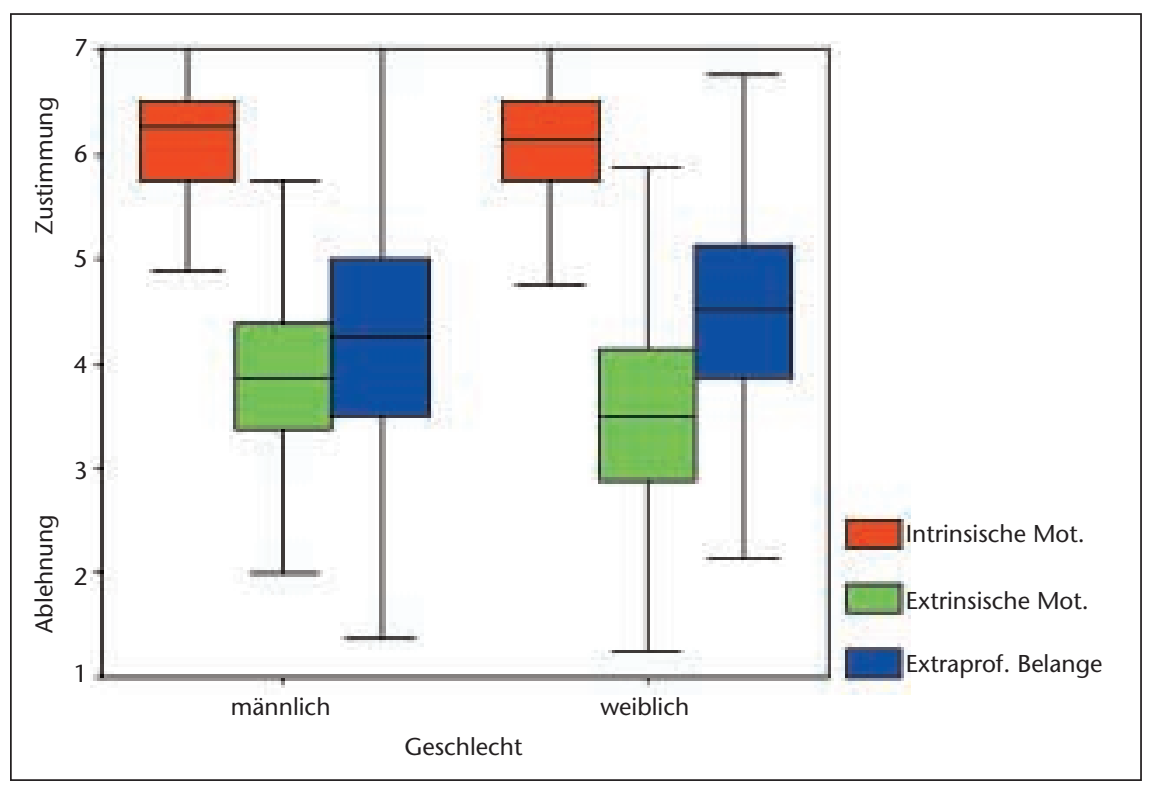

Anmerkung: Schattierte Box enthält $50 \%$ aller Fälle, horizontale Linie in Box entspricht Median, vertikale Linien indizieren höchsten und niedrigsten Wert. schlechtstypische Unterschiede. Ein Fachgebiet mit «technisch-apparativer Tätigkeit» oder ein Fachgebiet mit Möglichkeiten zu «Forschungstätigkeit» ist für mehr Studenten als Studentinnen wichtig, wohingegen Studentinnen häufiger ein Fachgebiet mit «intensivem Patientenkontakt» wählen. Die Aspekte «hohes Einkommen» und «hohes Ansehen» erachten angehende Ärzte signifikant häufiger als wichtig als Ärztinnen. Ärztinnen legen im Vergleich zu ihren männlichen Kollegen eher Wert auf die «Überschaubarkeit des Fachgebietes» und eine «kurze Ausbildungsdauer».

$56,3 \%$ der Studierenden, die einen Facharzttitel anstreben, haben sich bereits entschieden, welche Weiterbildung sie absolvieren wollen.

\section{Angestrebte berufliche Laufbahn}

Längerfristig ist die Mehrheit aller Befragten interessiert an einer Einzel- oder Gruppenpraxis $(71,9 \%)$ oder an einer klinischen Laufbahn (64,5\%). Ein Fünftel der Studienteilnehmenden zeigt Interesse an einer Universitätslaufbahn $(20,2 \%), 12,1 \%$ an einer Laufbahn in der Industrie (Mehrfachantwort).

\section{Berufliche Motivation}

Der Fragebogen zur beruflichen Motivation [9] misst drei Formen der beruflichen Orientierung. Die intrinsische Motivation erfasst Freude und Interesse an der beruflichen Tätigkeit selbst, die extrinsische Motivation das Interesse am beruflichen Aufstieg und die extraprofessionalen Belange das Interesse an anderen Lebensbereichen, insbesondere dem familialen im Vergleich zum beruflichen.

In Abbildung 2 sind die Verteilungen der Antworten auf den drei Motivationsskalen dargestellt. Alle Befragten schätzen ihre intrinsische Motivation als hoch ein. Die Antworten für die Skalen extrinsische Motivation und extraprofessionale Belange weisen eine grössere Streubreite auf, d.h. es gibt Studierende, die sich als hoch, und solche, die sich als niedrig auf diesen Dimensionen einschätzen. Die meisten Antworten fallen jedoch in den mittleren Bereich. Die Frauen zeigen gegenüber den Männern signifikant niedrigere Werte auf der Skala extrinsische Motivation $(\mathrm{p}<, 001)$, hingegen sind ihre Werte in bezug auf extraprofessionelle Belange höher $(\mathrm{p}<, 01)$. Keine signifikanten Unterschiede bestehen in der Skala intrinsische Motivation. 
Abbildung 3

Zufriedenheit mit verschiedenen Lebensbereichen und globale Lebenszufriedenheit (markiert durch roten Punkt) [13].

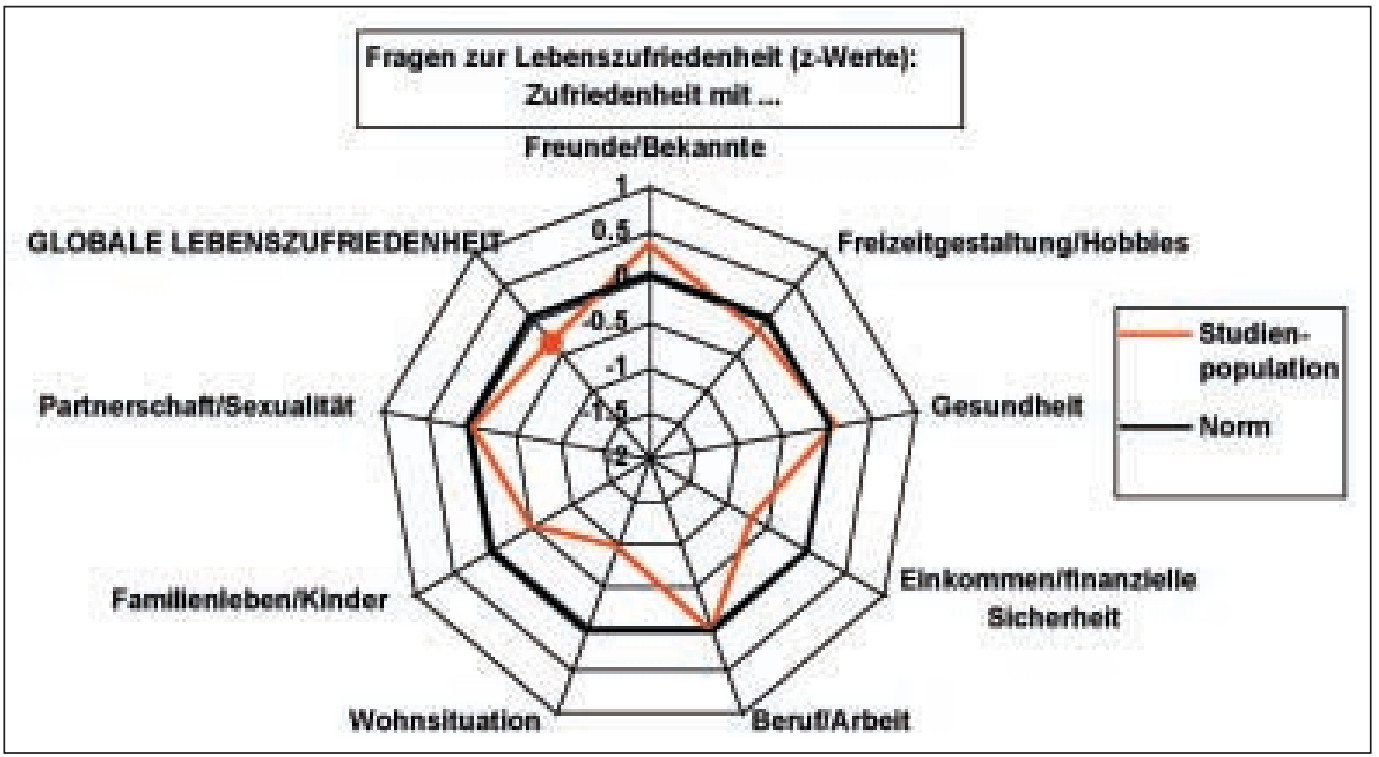

\section{Selbsteinschätzung}

\section{Selbstwertgefühl}

Das Selbstwertgefühl bezeichnet einen zentralen Aspekt des Selbstkonzeptes. Es umschreibt eine grundlegend positiv bzw. negativ getönte Einstellung sich selbst gegenüber. Mit der Rosenberg-Self-Esteem-Skala [10] wird das generelle Selbstwertgefühl erfasst. Durchschnittlich geben die Teilnehmenden auf dieser Skala einen Wert von 23,5 an (bei einem möglichen Maximum von 30 Punkten). Männer schätzen ihr Selbstwertgefühl signifikant höher ein als Frauen $(\mathrm{p}<, 05)$. Verglichen mit der Durchschnittspopulation haben angehende Ärztinnen und Ärzte jedoch ein hohes Selbstwertgefühl.

\section{Kohärenzgefühl}

Das Kohärenzgefühl [11] ist ein Mass für das Vertrauen in die eigene Fähigkeit, anfallende Entwicklungsaufgaben oder Stressoren zu bewältigen. In unserer Studie liegt der mittlere Wert auf dieser Skala bei 5,1 und damit geringfügig höher als in der Normpopulation. In unserer Befragung zeigen sich beim Kohärenzgefühl keine geschlechtstypischen Unterschiede, während in der Durchschnittsbevölkerung Männer in der Regel sowohl hinsichtlich ihres Selbstwert- wie auch ihres Kohärenzgefühls höhere Werte angeben.

\section{Persönlichkeitseigenschaften}

Der Personal Attributes Questionnaire (GE-PAQ) [12] misst für die Geschlechtsrolle charakteristische Persönlichkeitszüge auf den beiden Skalen
Handlungsorientierung und Gefühlsorientierung. Es zeigen sich signifikante geschlechtstypische Unterschiede, d.h. die männlichen Studierenden sind im Vergleich zu den weiblichen handlungsorientierter und die weiblichen Studierenden im Vergleich zu den männlichen gefühlsorientierter. Die Befragten beiderlei Geschlechts weisen jedoch auf beiden Skalen überdurchschnittlich hohe Werte auf. Dies bedeutet, dass junge Ärztinnen und Ärzte sowohl eine hohe Handlungsals auch eine hohe emotionale Orientierung im Vergleich mit der Durchschnittsbevölkerung aufweisen.

\section{Lebenszufriedenheit [13]}

Erfasst wird einerseits die Wichtigkeit verschiedener Lebensbereiche (Freunde, Freizeit, Gesundheit, Einkommen, Arbeit/Studium, Wohnen, Familie und Partnerschaft) und andererseits die Zufriedenheit der Befragten mit diesen Lebensbereichen. Im Wert der gewichteten Zufriedenheit werden Wichtigkeit und Zufriedenheit miteinander verrechnet. In die gewichtete Zufriedenheit fliesst so, neben der Zufriedenheit, auch die subjektive Bedeutung (Wichtigkeit) ein, welche eine Person den einzelnen Aspekten für ihre Lebensqualität beimisst (Abb. 3).

Der Mittelwert der gewichteten Zufriedenheit mit dem Aspekt Freunde ist bei den Befragten im Vergleich zur Allgemeinbevölkerung signifikant höher, die Befragten sind in diesem Bereich also zufriedener. Die gewichtete Zufriedenheiten mit den Aspekten Freizeit, Einkommen, Wohnen, Familie wie auch die globale Lebenszufriedenheit ist bei den befragten Personen jedoch 
signifikant niedriger als in der Durchschnittspopulation. In bezug auf die Aspekte Gesundheit, Beruf und Partnerschaft unterscheiden sich die angehenden Ärztinnen und Ärzte nicht von der Allgemeinbevölkerung.

\section{Wohlbefinden}

Ihr körperliches Wohlbefinden in den letzten 4 Wochen schätzen die Studierenden auf einer 7stufigen Skala durchschnittlich mit 5,5, ihr seelisches Wohlbefinden mit 5,3 Punkten ein. Einzig beim körperlichen Wohlbefinden zeigt sich ein signifikanter Geschlechtsunterschied, indem Frauen ihr Wohlbefinden im Durchschnitt als weniger gut bewerten als Männer.

\section{Diskussion}

Wie lassen sich die Angaben der angehenden Ärztinnen und Ärzte über ihre Studienerfahrungen und Karrierepläne interpretieren? Die hohe Teilnahmequote $(70 \%)$ deutet darauf hin, dass die unmittelbar vor dem Staatsexamen stehenden Studierenden sich intensiv mit Fragen ihres Studiums und ihrer beruflichen Zukunft beschäftigen. Viele fügten am Schluss des Fragebogens noch Bemerkungen an, dass diese Themen sowohl unter den Studierenden wie auch zwischen Studierenden und Dozierenden häufiger diskutiert werden sollten. Manche wünschten sich Informationsveranstaltungen zu den verschiedenen Karrieremöglichkeiten oder eine Mentorin/einen Mentor aus dem angestrebten Fachgebiet für Gespräche über ihre individuelle Berufsplanung.

Während bis vor 10 Jahren noch gut ein Drittel der Medizinstudierenden aus Arztfamilien stammten, kommt in unserer Studie nur noch jede/r siebte aus einem Medizinerelternhaus. Einerseits hat das Sozialprestige des Arztberufes in den letzten Jahren abgenommen. Andererseits könnte es sein, dass Ärzteeltern ihren Kindern aufgrund der Entwicklungen im Gesundheitswesen eher von einem Medizinstudium abraten.

Im Zusammenhang mit den angestrebten Reformen des Medizinstudiums, wonach ein Grossteil des Stoffes in Form von themen- und problemorientiertem Lernen vermittelt werden soll, interessierte in unserer Studie, welche Erfahrungen die Studierenden in den Unterassistenzen und klinischen Praktika gemacht haben. Nach den vielen Magistralvorlesungen und der Vermittlung von mehrheitlich naturwissenschaftlich-theoretischem Wissen schätzten sie, im zweiten Teil des Studiums endlich klinische Erfahrungen sammeln zu können. Deshalb fällt die Gesamteinschätzung mehrheitlich positiv aus, obwohl in den Kommentaren der Teilnehmenden bemängelt wurde, dass in vielen Kliniken ein Ausbildungskonzept für die Unterassistenz fehlt. Die fachliche Anleitung erhielt nur eine durchschnittliche Bewertung. Diejenigen, welche einige Monate als Unterassistierende in den USA arbeiteten, lobten vor allem das sehr gute und strukturierte «Teaching» an den dortigen Kliniken.

Bis zum Inkrafttreten der Bilateralen Verträge (1. Juni 2002) und bis zum darauf aufbauenden Freizügigkeitsgesetz (FMPG) wurde eine Dissertation als obligatorischer Bestandteil für den Erwerb eines Facharzttitels verlangt. Vier Fünftel der Teilnehmenden - deutlich mehr Frauen als Männer - haben ihre Dissertation im 6. Studienjahr entweder bereits abgeschlossen oder in Bearbeitung. Es ist zu vermuten, dass Medizinerinnen unter Berücksichtigung ihres Wunsches, eine Familie zu gründen, ihre Weiterbildung zielstrebiger planen und deshalb auch die Dissertation möglichst schon während des Studiums abschliessen wollen.

Mit der zunehmenden Spezialisierung innerhalb der Medizin, der Reglementierung der ärztlichen Weiterbildung und dem in manchen Fachgebieten ungünstigen Verhältnis zwischen Angebot von und Nachfrage nach Stellen sehen sich die Studierenden gezwungen, bereits während der Unterassistententätigkeit Weiterbildungsstellen an entsprechenden Kliniken zu vereinbaren. Die Hälfte der Befragten hat zu Beginn des 6. Studienjahres eine oder mehrere Stellen abgemacht. Knapp zwei Drittel wissen zu diesem Zeitpunkt auch schon, in welchem Fachgebiet sie sich spezialisieren wollen. Bei der Wahl des Fachgebietes zeigen sich in unserer wie auch in anderen Studien [14] geschlechtstypische Unterschiede. Angehende Ärztinnen wählen Fachgebiete, in denen beziehungsorientierte Aspekte der ärztlichen Tätigkeit eine grosse Rolle spielen, während angehende Ärzte eher handlungsorientierte Disziplinen bevorzugen [15]. Da Frauen mehrheitlich einen Lebensentwurf planen, der die Option einer Familie beinhaltet, berücksichtigen sie auch Ausbildungsdauer und Überschaubarkeit des Fachgebietes. Die meisten Medizinerinnen möchten ihre Facharztweiterbildung abschliessen, bevor sie eine Familie gründen. Obwohl die angehenden Ärztinnen und Ärzte unserer Studie in gleicher Weise Wert auf die Vereinbarkeit von Beruf und Familie legen, zeigt sich in unserer wie in anderen Untersuchungen [16], dass bei Männern im Gegensatz zu Frauen der Wunsch nach einer Familie ihre Karrierepläne nicht tangiert. 
In den kommenden Jahren wird die Hälfte der Fachärzte Frauen sein. Wenn dieses Potential gut ausgebildeter Ärztinnen dem Gesundheitswesen nicht verlorengehen soll, müssen zur Vereinbarkeit von Beruf und Familie neue Wege gefunden werden. Dringend erforderlich in dieser Hinsicht sind der Ausbau von Kinderbetreuungsmöglichkeiten, flexiblere Arbeitszeitmodelle, Unterstützung beim Wiedereinstieg nach einem Mutterschaftsurlaub und strukturierte Programme zur Nachwuchsförderung, die auf die speziellen Bedürfnisse von Frauen mit familiären Verpflichtungen zugeschnitten sind. Am UniversitätsSpital Zürich werden solche Massnahmen derzeit im Rahmen eines Projekts zur gleichberechtigten Nachwuchsförderung umgesetzt.

Sowohl das Medizinstudium wie auch die spätere ärztliche Tätigkeit verlangen einen hohen persönlichen Einsatz. Von daher überrascht es nicht, dass Personen, die sich für den Arztberuf entscheiden, über ein vergleichsweise hohes Selbstwert- und Kohärenzgefühl verfügen. In der Durchschnittsbevölkerung geben Männer in beiden Bereichen höhere Werte an [17]. Interessanterweise schätzen sich aber angehende Ärztinnen hinsichtlich ihres Kohärenzgefühls ähnlich hoch ein wie ihre männlichen Kollegen. Es ist zu vermuten, dass sich überwiegend Frauen, die ein grosses Vertrauen in ihre Fähigkeit haben, anfallende Belastungen zu bewältigen, für den Arztberuf entscheiden. Die meisten Frauen, welche einen hohen ideellen, zeitlichen und materiellen Einsatz in ihre Ausbildung investieren und während vieler Jahre persönliche Bedürfnisse zurückstellen, möchten später sowohl ihren Beruf ausüben als sich auch für eine Familie engagieren. Viele Medizinstudentinnen fassen also eine spätere Doppelbelastung von Beruf und Familie ins Auge. Andererseits zeigt sich bei der Einschätzung des aktuellen körperlichen und seelischen Wohlbefindens, dass Frauen ihr körperliches Wohlbefinden als weniger gut bewerten als ihre männlichen Kollegen. Möglicherweise fühlen sich Medizinstudentinnen durch die Anforderungen des Studiums in Kombination mit persönlichen Aufgaben, Verpflichtungen und Interessen körperlich mehr belastet. Ähnliche Ergebnisse berichten auch Brunner \& Bachmann [18]: Medizinstudentinnen geben schon vor Beginn des Studiums und dann im Verlauf des Studiums noch zunehmend mehr körperliche Beschwerden im Vergleich mit anderen Studierenden an.

Die Angaben aus den Fragebogen deuten darauf hin, dass ein tragfähiges soziales Umfeld und verlässliche Freundesbeziehungen zu den positivsten Erfahrungen während des Studiums zählen.
Viele Studierende äussern, dass sie nur dank dieser Unterstützung die Belastungen des Studiums hätten bewältigen können. In einer Untersuchung zur Bedeutung von Belastung und Ressourcen für die Gesundheit der Studierenden der Universität und ETH Zürich (RUBIS-Studie) [19] zeigen die Ergebnisse ebenfalls, dass die soziale Unterstützung durch Mitstudierende die wichtigste Ressource für die Gesundheit darstellt. In anderen für die Lebenszufriedenheit wichtigen Bereichen wie Freizeit, Einkommen, Wohnen und Familie weisen die Befragten erwartungsgemäss eine geringere Zufriedenheit auf. Die angehenden Ärztinnen und Ärzte wissen, dass sich die Beanspruchung in den ersten Jahren ihrer Assistenzarzttätigkeit noch verstärken wird [20]. Von daher blicken sie eher skeptisch in die $\mathrm{Zu}$ kunft und zweifeln daran, dass sich ihre Lebenszufriedenheit bessern könnte. Ähnliche Einschätzungen berichten auch andere Autoren $[6,21,22]$. Die Befragten unserer Studie freuen sich auf den Einstieg in den ärztlichen Beruf, sind sich aber der Auswirkungen bewusst, welche ihr berufliches Engagement auf ihr Privatleben haben. Vor allem die Medizinerinnen sehen sehr realistisch, wie schwierig es für sie werden wird, eine Balance zwischen ihren persönlichen und beruflichen Lebenszielen zu finden. Von daher formulieren sie eher umschriebene und ihnen realisierbar erscheinende Karrierepläne.

\section{Ausblick}

Trotz vieler kritischer Äusserungen und Befürchtungen bezüglich ihrer beruflichen Zukunft haben angehende Ärztinnen und Ärzte eine hohe Berufsmotivation. Wie auch von Abele [6] berichtet, ist für Mediziner beiderlei Geschlechts das Idealbild des Arztes bzw. der Ärztin humanistisch, fürsorglich und beziehungsorientiert. Allerdings erleidet das beziehungsorientierte Ideal während des praktischen Jahres einen deutlichen «Dämpfer» [6]. Ärztliche Wertvorstellungen verändern sich: Beziehungsorientierte Idealvorstellungen, in denen Kommunikation und psychosoziale Kompetenz im Mittelpunkt der ärztlichen Tätigkeit stehen, werden durch die Realität des Klinikalltags relativiert. Die Assistierenden werden nüchterner und pragmatischer. Wie erwähnt sollen die Studienteilnehmenden während ihrer Weiterbildungsjahre in zweijährigen Intervallen erneut befragt werden. Die Ergebnisse der Längsschnittstudie sollen Aufschluss über karrierefördernde und -behindernde Faktoren sowohl aus persönlichen als auch institutionellen Gründen geben. 


\section{Dank}

Herrn lic. phil. Steffen Niemann danken wir für die sehr kompetente Adressverwaltung im Rahmen des Forschungsprojekts.

\section{Literatur}

1 Bundesamt für Statistik. Statistik zu Studierenden der Humanmedizin in der Schweiz. Bern: Sektion Hochschulen und Wissenschaft; 2002.

2 Generalsekretariat der Verbindung der Schweizer Ärztinnen und Ärzte. FMH-Ärztestatistik. Schweiz Ärztezeitung 2002;83(18):871-90.

3 Buddeberg-Fischer. B. Karriereentwicklungen von Frauen und Männern in der Medizin. Schweiz Ärztezeitung 2001;82:1838-44.

4 Abele A. Lebens- und Berufsplanung von Frauen. In: Roos-Schuhmacher H (Hrsg.). Kompetent in die Öffentlichkeit. Frauen auf dem Weg in die BürgerInnen-Gesellschaft. Opladen: Leske \& Budrich; 2001. S. 27-43.

5 Sieverding M. Psychologische und soziale Karrierehemmnisse im Berufsweg der Ärztin. Arch Gynecol Obstetr 1995;257:699-704.

6 Abele AE. Arzt oder Ärztin sein heute - zwischen Erwartung und Realität. Befunde der Erlanger Längsschnittstudie BELA-E. In: Brandenburg U, Leeners B, Petermann-Meyer A, Schwarte A, Dohmen C, Neises M (Hrsg.). Psychosomatische Gynäkologie und Geburtshilfe. Beiträge der Jahrestagung 2001 der DGPFG. Giessen: Psychosozial; 2002. S. 21-31.

7 Abele A, Andrae M, Schute M. Wer hat nach dem Hochschulexamen schnell eine Stelle? Erste Ergebnisse der Erlanger Längsschnittstudie (BELA-E). Z Arbeits Organisationspsychol 1999; 43:95-101.

8 Hoff E, Grote S, Hohner HU, Dettmer S. Berufsverlaufsmuster und Geschlecht in Medizin und Psychologie. Z Polit Psychol 2000;8:203-23.

9 Abele AE. Karriereorientierungen angehender Akademikerinnen und Akademiker. Bielefeld: Kleine; 1994.

10 Rosenberg M. Society and the adolescent selfimage. Princeton N.J.: University Press; 1965. Deutsche Version des Fragebogens zum Selbstwertgefühl: Filipp SH, Ferring D, Freudenberg E, Klauer T. Affektiv-motivationale Korrelate von Formen der Krankheitsbewältigung: Erste Ergebnisse einer Längsschnittstudie mit Krebspatienten. Psychother Med Psychol 1988;38:37-42.

11 Antonovsky A. Unraveling the mystery of health. How people manage stress and stay well. San Francisco: Jossey-Bass; 1987. Deutsche Version: Abel T, Kohlmann, T. Der Fragebogen zum Kohärenzsinn. In: Brähler E, Schumacher J, Strauss B (Hrsg.). Diagnostische Verfahren in der Psychotherapie. Göttingen: Hogrefe; 2002. S. 326-30.
12 Alfermann D. Androgynie. In: Reigber D (Hrsg.). Frauen-Welten. Düsseldorf: Econ; 1993. S. $147-200$.

13 Henrich G, Herschbach P. Questions on Life Satisfaction $\left(\mathrm{FLZ}^{\mathrm{M}}\right)$. A short questionnaire for assessing subjective quality of life. Eur J Psychol Assess 2000;16:150-59.

14 Gjerberg E. Medical women - towards full integration? An analysis of the specialty choices made by two cohorts of Norwegian doctors. Soc Sci Med 2001;52:331-43.

15 Sieverding M. Geschlechts(rollen-)unterschiede im ärztlichen Beruf. In: Brähler E, Strauss B (Hrsg.). Handlungsfelder der Psychosozialen Medizin. Göttingen: Hogrefe; 2002. S. 117-34.

16 Lambert T, Goldacre MJ. Career destinations seven years on among doctors who qualified in the United Kingdom in 1988: Postal questionnaire survey. Br Med J 1998;317:1429-31.

17 Schumacher J, Gunzelmann T, Brähler E. Deutsche Normierung der Sense of Coherence Scale von Antonovsky. Diagnostica 2000; 46(4):208-13.

18 Brunner S, Bachmann N. Psychische und physische Gesundheit im Verlauf des Studiums. In: Bachmann N, Berta D, Eggli P, Hornung R (Hrsg.). Macht Studieren krank? Die Bedeutung von Belastung und Ressourcen für die Gesundheit der Studierenden. Bern: Huber; 1999. S. 77-92.

19 Bachmann N. Der Einfluss des Studiums und der damit verbundenen Lebensumstände auf die Gesundheit der Studierenden. In: Bachmann N, Berta D, Eggli P, Hornung R (Hrsg.). Macht Studieren krank? Die Bedeutung von Belastung und Ressourcen für die Gesundheit der Studierenden. Bern: Huber; 1999. S. 155-69.

20 Stern K. Ende eines Traumberufs? Lebensqualität und Belastungen bei Ärztinnen und Ärzten. Münster, New York: Waxmann; 1996.

21 Sieverding M. Berufskonzepte von Medizinstudierenden: Kongruenzen und Diskrepanzen zwischen Selbstkonzept, beruflichem Idealkonzept und Karrierekonzept. Z Arbeits Organisationspsychol 1992;36:157-66.

22 Jurkat H, Reimer C, Schröder K. Erwartungen und Einstellungen von Medizinstudentinnen und -studenten zu den späteren Belastungen und Folgen ihrer späteren ärztlichen Tätigkeit. Psychother Psychosom Med Psychol 2000;50:215-21. 